Original Article

\title{
Comparison of spatiotemporal gait parameters with a spinal orthosis and without a spinal orthosis on level ground and stairs
}

\author{
Hyeon-Nam Song, PhD, $\mathrm{PT}^{1)}$, Young Mi Kim, PhD, $\mathrm{PT}^{1)}$, Kyoung Kim, PhD, $\mathrm{PT}^{2)^{*}}$ \\ 1) Department of Rehabilitation Science, Graduate School, Daegu University, Republic of Korea \\ 2) Department of Physical Therapy, College of Rehabilitation Science, Daegu University: \\ 201 Daegudae-ro, Jillyang, Gyeongsan-si, Kyeongbuk 712-714, Republic of Korea
}

\begin{abstract}
Purpose] This study attempted to examine the impact on the pattern of walking on both level ground and stairs after restricting the movement of the spine and the trunk of the body by using a spinal orthosis. [Subjects and Methods] Forty healthy males in their 20s were selected as the sample, which was randomly and evenly divided into two groups: (1) the WT group (with a thoracolumbosacral orthosis group) and (2) the WOT group (Without a thoracolumbosacral orthosis group). The spinal orthosis used in this study was a thoracolumbosacral orthosis called a plastic body jacket. [Results] In walking on level ground, step width showed a significant difference, but no statistically significant difference was found between the WT group and the WOT group with respect to the other variables. In walking on stairs, the double stance phase showed a statistically significant difference during stair descent in the WT group compared with the WOT group. [Conclusion] It was proven that wearing a TLSO increased walking stability in the case of walking on level ground and stairs. Hence, it is believed that proper use of a spinal orthosis can play an ancillary role in daily life and therapeutic interventions.

Key words: Thoracolumbosacral orthosis, Motion analysis, Spatiotemporal variables
\end{abstract}

(This article was submitted Feb. 22, 2016, and was accepted Apr. 7, 2016)

\section{INTRODUCTION}

The spine functions usefully during walking as a tool for maintaining upright posture and balance ${ }^{1)}$. If deformation of the spine occurs, the ability to compensate for the posture change is lost, and the resulting imbalance of the trunk of the body induces clinically recognizable walking deviation. Due to the influence on the stability of the trunk of the body and the general musculoskeletal system, dysfunction in them can also induce pain and spinal disability2).

A thoracolumbosacral orthosis (TLSO), a brace that extends from the spine to the pelvis, has a direct impact on the movement of the spine during walking and hence has a significant influence on the movement of the pelvis and the lower limbs. Spinal bracing is used in a variety of acute and chronic conditions. The efficacy of bracing of the lumbar spine is thought to be the result of a complex interaction of several factors, including but not limited to motion reduction, increased intra-abdominal pressure, and pressure feedback on the musculature ${ }^{3)}$. Therefore, this study attempted to examine the impact on the pattern of walking on both level ground and stairs after restricting the movement of the spine and the trunk of the body by using a spinal orthosis.

\section{SUBJECTS AND METHODS}

In this study, 40 healthy males in their 20 s were selected as a sample, which was randomly and evenly divided into two

\footnotetext{
*Corresponding author. Kyoung Kim (E-mail: kykim257@hanmail.net)

(C2016 The Society of Physical Therapy Science. Published by IPEC Inc.

This is an open-access article distributed under the terms of the Creative Commons Attribution Non-Commercial No Derivatives (by-nc-nd) License $<$ http://creativecommons.org/licenses/by-nc-nd/4.0/>.
} 
groups: (1) the WT Group (with TLSO group) and (2) the WOT group (Without a TLSO group). The inclusion criteria included (1) no neurologic disease history, (2) capability of maintaining independent standing, walking, and landing, (3) no surgical history that could affect walking or landing, and (3) no inborn deformity or disease in the relevant areas, including the foot, pelvis, spine, etc.

For the WT group, the average age was $24.9 \pm 2.4$ years, the average height was $173.3 \pm 4.2 \mathrm{~cm}$, and the average weight was $70.6 \pm 6.6 \mathrm{~kg}$. For the WOT group, the average age was $25.3 \pm 2.7$ years, the average height was $175.6 \pm 4.4 \mathrm{~cm}$, and the average weight was $69.1 \pm 6.8 \mathrm{~kg}$. Using Levene's variance homogeneity test and a normality test on the general physical characteristics, no statistically significant difference was found between the two groups $(\mathrm{p}>0.05)$. All of the patients included understood the purpose of this study and provided their written informed consent prior to participation in accordance with the ethical principles of the Declaration of Helsinki.

The spinal orthosis used in this study was a TLSO called a plastic body jacket. It is made of lightweight polypropylene that has thermoplasticity, and it is designed so that it is easy to put on and take off. It is a ready-made product that comes in four sizes (extra-large, large, medium, and small), and the appropriate size was used for the body figure of the research subjects. The plastic body jacket is known to restrict the movement in three 3-dimensional planes more effectively than any other TLSO, including (1) flexion and extension of the body's trunk in the sagittal plane, (2) lateral flexion in the frontal plane, and (3) rotation of the trunk of the body in the horizontal plane. It is also known to be most effective in maintaining the body's alignment.

The steps of the staircase fabricated and use in this study had a height of $17 \mathrm{~cm}$, width of $28 \mathrm{~cm}$, and breadth of $61 \mathrm{~cm}$ and were fabricated according to the housing construction standards; four steps were installed for this study. A motion analysis system composed of six infrared cameras (Vicon motion capture system, Vicon Motion Systems Ltd., Oxford, UK) was used for measurement of spatiotemporal variables and for the kinematic variables of walking, with the sample collection rate of the infrared camera set at $120 \mathrm{~Hz}$.

The subjects were told to walk comfortably and naturally down a $10 \mathrm{~m}$ long straight walking lane drawn on firm, even ground at their usual speed while looking straight ahead. Walking on level ground was performed after oral instruction, and this was followed by ascending and descending the stairs. The measurement interval was not mentioned so that the subjects would not recognize when the measurements began. Walking was measured after the first five footsteps, and a 30-second rest period was given after each motion.

\section{RESULTS}

The mean and average values of the spatiotemporal variables of the WT group and the WOT group while walking on a level ground are shown below. The step width revealed a significant difference $(\mathrm{p}<0.05)$, but no statistically significant difference was found between the WT group and the WOT group with respect to the other variables (p>0.05) (Table 1). The mean and average values of the spatiotemporal variables of the WT group and the WOT group during walking on stairs are also shown below. In the double stance phase during stair descent, there was a statistically significant difference between the WT group and the WOT group $(\mathrm{p}<0.05)$ (Table 1$)$.

\section{DISCUSSION}

This study attempted to investigate the impact of wearing a TLSO on the movement of the lower limbs during walking. Spatiotemporal variables were compared and analyzed in the cases of walking on level ground and stairs, which are

Table 1. Comparison of spatioteporal variables between the WT group and the WOT group

\begin{tabular}{llcc}
\hline Gait condition & Parameters & WOT group & WT group \\
\hline \multirow{5}{*}{ Level ground } & Cadence (steps/min) & $113.8 \pm 8.6$ & $113.5 \pm 9.1$ \\
& Step length (cm) & $67.3 \pm 4.8$ & $68.9 \pm 6.2$ \\
& Step width (cm)* & $13.9 \pm 2.2$ & $12.7 \pm 1.4$ \\
& Stride length (cm) & $133.7 \pm 6.7$ & $135.0 \pm 10.6$ \\
& Walking speed (cm/sec) & $127.8 \pm 14.6$ & $130.2 \pm 14.7$ \\
Stair ascent & Cycle duration (sec) & $1.4 \pm 0.2$ & $1.5 \pm 0.2$ \\
& Stance phase (\%) & $65.8 \pm 4.5$ & $67.5 \pm 6.9$ \\
& Double stance phase (\%) & $14.7 \pm 1.9$ & $14.2 \pm 2.9$ \\
Stair descent & Cycle duration(sec) & $1.2 \pm 0.0$ & $1.2 \pm 0.0$ \\
& Stance phase (\%) & $62.1 \pm 3.2$ & $63.9 \pm 6.5$ \\
& Double stance phase (\%)* & $13.1 \pm 1.8$ & $1.8 \pm 2.1$ \\
\hline
\end{tabular}

*Significantly different between with a TLSO and without a TLSO $(\mathrm{p}<0.05)$.

WT group: with a TLSO group; WOT group: without a TLSO group; TLSO: thoracolumbosacral orthosis 
frequently performed in daily life.

With respect to the spatiotemporal variables, the measurements differed between walking on level ground and walking on stairs as a result of the spatiotemporal characteristics of walking, which can change due to step height, step width, and grade of the staircase. For walking on level ground, there was no significant difference in cadence, step length, stride length, or walking speed between the WT group and the WOT group. It is believed that BOS (base of support) for maintaining balance decreased as walking stability improved as a result of wearing the spinal orthosis, as was also reported by Schmidt et $\mathrm{al}^{4}$. Meanwhile, Konz et al. ${ }^{2}$ ) revealed that cadence remarkably increased when wearing a plastic body jacket, although walking speed and step length did not display any difference. Moreover, step length was also shortened overall, although the difference was not statistically significant. It can be interpreted that the walking speed remained constant because the cadence increased due to the decreased rotation angle of the pelvis in the parallel plane, but that the step length decreased to compensate for other factors.

In a comparison of the spatiotemporal variables during walking on stairs, there was no significant difference in the case of stair ascent, but the double stance phase significantly decreased in the case of stair descent. Walking on stairs also reduces stability by increasing head and neck movements ${ }^{5}$. Because restricting movement of the trunk of the body during stair descent can cause the upper body to remain straight and enable the center of weight to be maintained toward the back of the basal area, it can ensure walking stability. Meanwhile, the level of tilt of the upper body can be checked by looking at the change in pelvic tilt during walking. During stair ascent, the body not only moves forward but also simultaneously moves upward.

To explain these differences, Kim et al. ${ }^{6}$ argued that the pelvis tilts forward when walking up stairs because tilting of the upper body forward from a straight standing position should shift the center of weight forward. On the contrary, in the case of stair descent in this study, stability was best maintained by preventing the upper body from leaning forward and maintaining the center of weight on or behind the basal area while keeping the upper body straight and minimizing rotating body movement. Clark and Phillips ${ }^{7}$ argued that the double stance phase can be an indirect index for checking lower limb stability and body balance. Stair ascent and descent demand more effort compared with walking on level ground, since upward and forward forces are required to support the body's weight and the continuous ability to control limits of stability is also required ${ }^{8}$.

One of the limitations of this study is that the TLSO used was a commercial product. This means that the restriction range for the trunk of the body could be different if a custom orthosis is used or the material is changed. Moreover, the sample size was small, and it does not adequately represent those with spinal lesions, as the research subjects consisted of young, healthy males only.

In conclusion, it was proven that wearing a TLSO increased the walking stability in the case of walking on level ground and walking on stairs. Hence, it is believed that proper use of a spine orthosis can play an ancillary role in daily life and therapeutic interventions.

\section{ACKNOWLEDGEMENT}

This research was supported by a Daegu University Research Grant in 2013.

\section{REFERENCES}

1) Feipel V, De Mesmaeker T, Klein P, et al.: Three-dimensional kinematics of the lumbar spine during treadmill walking at different speeds. Eur Spine J, 2001, 10: 16-22. [Medline] [CrossRef]

2) Konz R, Fatone S, Gard S: Effect of restricted spinal motion on gait. J Rehabil Res Dev, 2006, 43: 161-170. [Medline] [CrossRef]

3) Vander Kooi D, Abad G, Basford JR, et al.: Lumbar spine stabilization with a thoracolumbosacral orthosis: evaluation with video fluoroscopy. Spine, 2004, 29: 100-104. [Medline] [CrossRef]

4) Schmidt K, Hübscher M, Vogt L, et al.: [Influence of spinal orthosis on gait and physical functioning in women with postmenopausal osteoporosis]. Orthopade, 2012, 41: 200-205. [Medline] [CrossRef]

5) Kim YM, Lee SY, Han JT, et al.: Kinematic analysis of head and trunk of elderly subjects during climbing of stairs and a ramp. J Phys Ther Sci, 2013, 25: 441-444. [CrossRef]

6) Kim YS, Kim EJ, Seo CJ: The comparative analysis of EMG and Gait Patterns of Lower extremities during going up stairs and down. Kor J Phys Edu, 2006, 45: 535-545.

7) Clark JE, Phillips SJ: A longitudinal study of intralimb coordination in the first year of independent walking: a dynamical systems analysis. Child Dev, 1993, 64: 1143-1157. [Medline] [CrossRef]

8) Lee J, Seo K: The effects of stair walking training on the balance ability of chronic stroke patients. J Phys Ther Sci, 2014, 26: 517-520. [Medline] [CrossRef] 